\title{
15. PALEOMAGNETIC AND ROCK MAGNETIC PROPERTIES OF HOLE 896A ${ }^{1}$
}

\author{
Simon Allerton, ${ }^{2}$ Horst-Ulrich Worm, ${ }^{3}$ and Laura B. Stokking ${ }^{4}$
}

\begin{abstract}
Ocean Drilling Program Hole 896A was drilled $1 \mathrm{~km}$ southeast of the ODP deep-basement Hole 504B, $200 \mathrm{~km}$ south of the Costa Rica Rift. The magnetic properties (intensity of remanence, bulk susceptibility, inclination of stable remanence, and median destructive field) of 166 minicores from Hole 896A are presented. The basement in Hole 896A can be divided into three sections on the basis of its magnetic properties. The upper part, to $330 \mathrm{~m}$ below seafloor (mbsf), has relatively high intensity of remanence $\left(J_{0}\right)$ and low susceptibility values. The middle section, between 330 and $360 \mathrm{mbsf}$, has high $\left(J_{0}\right)$ and intermediate susceptibility values, and the lower section, below $360 \mathrm{mbsf}$, has low $\left(J_{0}\right)$ and high susceptibility values. The magnetic mineralogy was investigated by monitoring the effect of heating on susceptibility. These results suggest that titanomaghemites occur throughout the hole and that titanomagnetites occur mainly below $330 \mathrm{mbsf}$. Some samples have evidence for minor amounts of hematite. The main cause of variation in the susceptibility and the median destructive field can be related to grain size. Grain size also controls $J_{0}$, although the degree of maghematization and the remanence mechanism of the magnetization also contribute. The magnetic properties of the upper part of Hole 896A, above the massive flow Unit 24, correlate with the upper part of Hole 504B above Unit 2D, at depths of 3796 and $3773 \mathrm{~m}$ below sea level, respectively. We suggest that the upper section corresponds to a late volcanic pile extruded onto a ponded flow that masks the earlier basement topography. This flow has probably acted as a major barrier to hydrothermal circulation. These results have significant implications for the interpretation of magnetic field data from ridges. The uppermost layer of late extrusive rocks has a high magnetization and a highly variable thickness, and may be an important contributor to variations in the magnetic signal at ridges.
\end{abstract}

\section{INTRODUCTION}

Our understanding of the magnetization of the oceanic crust comes largely from Deep Sea Drilling Project (DSDP) and Ocean Drilling Program (ODP) drilling (e.g., Furuta and Levi, 1983; Smith and Banerjee, 1986; Pariso and Johnson, 1991) and from ophiolites (e.g., Vine and Moores, 1972; Banerjee, 1980). Within these studies, ODP Hole 504B holds a special place as the deepest hole in ocean basement, penetrating $1.8 \mathrm{~km}$ of oceanic basement, including more than $1 \mathrm{~km}$ of sheeted dikes. These studies, particularly those of DSDP/ODP drill holes, by their nature lead to a one-dimensional layered model of the magnetic properties of the oceanic crust. ODP Hole $896 \mathrm{~A}$ was drilled $1 \mathrm{~km}$ southeast of Hole 504B and provides an opportunity to test the validity of these layered models. In this chapter, we describe the rock magnetic properties of Hole 896A, discuss their origin, and compare them with those of DSDP/ODP Hole 504B.

\section{GEOLOGICAL SETTING AND DRILLING OF HOLE 896A}

Hole $896 \mathrm{~A}$ is located at $1^{\circ} 13.006^{\prime} \mathrm{N}, 83^{\circ} 43.392^{\prime} \mathrm{W}$, about $1 \mathrm{~km}$ southeast of Hole 504B (Fig. 1) and about $200 \mathrm{~km}$ south of the spreading axis of the Costa Rica Rift (Alt, Kinoshita, Stokking, et al., 1993). It was drilled on a bathymetric high over a basement high observed on single-channel seismic reflection data, in a water depth of $3448 \mathrm{~m}$ below sea level (mbsl). Basement was reached at $179 \mathrm{~m}$ below seafloor (mbsf) and was cored from 195 to $469 \mathrm{mbsf}(274 \mathrm{~m})$, with a recovery of about $74 \mathrm{~m}$ of rock. Basement is composed of ex-

'Alt, J.C., Kinoshita, H., Stokking, L.B., and Michael, P.J. (Eds.), 1996. Proc, ODP, Sci. Results, 148: College Station, TX (Ocean Drilling Program).

${ }^{2}$ Department of Geology and Geophysics, University of Edinburgh, The Grant Institute, West Mains Road, Edinburgh EH9 3JW.United Kingdom. simona@glg.ed.ac.uk

${ }^{3}$ Bundesanstalt für Geowissenschaften und Rohstoffe, Stilleweg 2, Postfach 510153, D-30631 Hannover, Federal Republic of Germany.

${ }^{4}$ Ocean Drilling Program, Texas A\&M University Research Park, 1000 Discovery Drive, College Station, TX 77845-9547, U.S.A. stokking@ cook.tamu.edu trusive and shallow intrusive lithologies including pillow lavas $(57 \%)$, massive units (38\%), breccias (5\%), and two small dikes. The volcanic section can be divided into upper and lower sections. The upper section (195-390 mbsf) is dominantly plagioclase-olivine phyric, and the lower section (390-469 mbsf) is commonly plagioclaseolivine-clinopyroxene phyric.

The ages of Sites 896 and 504 have been determined from their position relative to the magnetic anomaly time scale. Hobart et al. (1985) identified Site 504 as 70\% across the 5.62-6.06-Ma reversal, according to the LaBrecque et al. (1977) time scale, which corresponds to an age of $5.93 \mathrm{Ma}$. Site 896 lies at a position $85 \%$ across this reversal, giving an age of $5.99 \mathrm{Ma}$. Updated time scales (e.g., Cande and Kent, 1992) now place this reversed period in the interval 6.376-6.744 Ma, yielding revised ages of $6.63 \mathrm{Ma}$ for Site 504 and 6.68 Ma for Site 896. The Costa Rica Rift has an intermediate spreading rate $(3.6 \mathrm{~cm} / \mathrm{yr}$ half-rate $)$ and a relatively simple crustal structure with a half-graben tilted away from the ridge, as imaged on singlechannel seismic reflection profiles (Langseth et al., 1988).

\section{MEASUREMENT OF MAGNETIC PROPERTIES}

This study is based on measurements from 71 shipboard samples and from shore-based studies of 66 samples in Oxford, United Kingdom, samples) and 29 samples at BGR, Grubenhagen, Germany. Of these 166 samples, $87(52 \%)$ are from pillow flows, $77(47 \%)$ are from massive flows, and $2(1 \%)$ are from breccias. The sample set thus slightly underrepresents the pillow flows and breccias and overrepresents the massive flows in the recovered core. As recovery is only about $27 \%$, the actual proportions of these lithologies in the drilled section may be significantly different. Because pillows and breccias tend to break up during drilling, these lithologies are probably significantly underrepresented in the core and in this study.

Properties measured include the measurement of the direction and intensity of natural remanence, bulk susceptibility, acquisition of isothermal remanent magnetization (IRM), and variation of susceptibility with temperature (Table 1). The intensity of remanence and the volume susceptibility are calculated using volumes derived using 


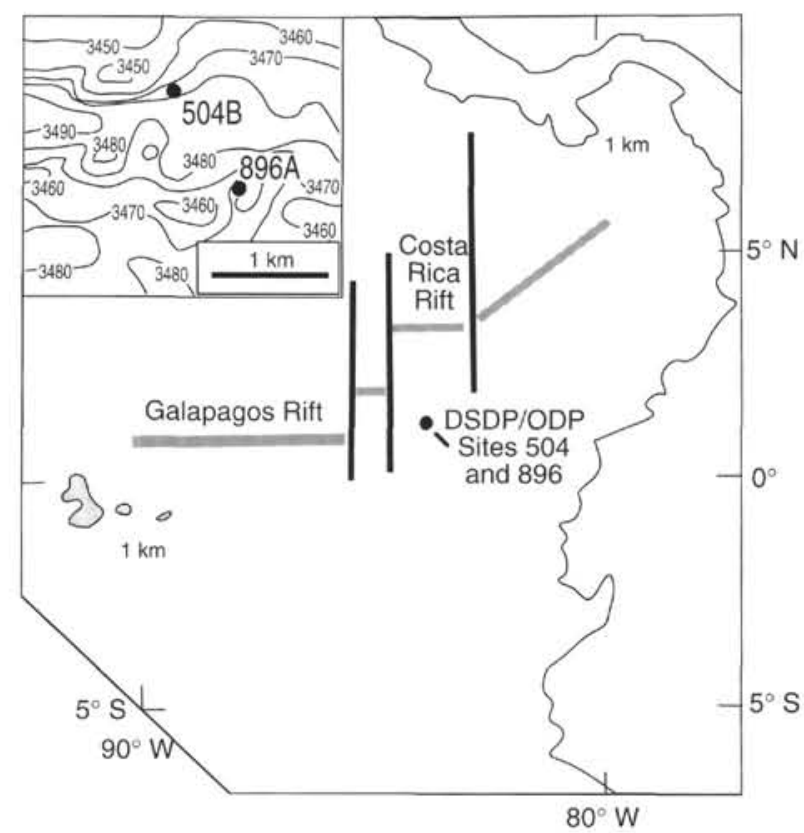

Figure I. Location of DSDP/ODP Sites 504 and 896 south of the Costa Rica Rift in the eastern equatorial Pacific (modified from Hobart et al.. 1985). Bathymetric contours in kilometers. Inset: Detailed bathymetry (in meters) at Hole 504B and 896A.

either Archimedes' principle (shipboard samples) or a simple geometrical estimate (Oxford and BGR).

The magnetic properties reported here are essentially similar to those described in Alt, Kinoshita, Stokking, et al. (1993), but are based on a larger sample set. Because the cores are free to rotate in the barrel, the declinations are measured relative to an arbitrary "working" reference frame, rather than to an in situ reference, so only the inclination can be obtained from these partially oriented samples.

The various magnetic properties discussed in detail below show a variation with depth. Significant changes are noted at about $330 \mathrm{mbsf}$ (top of Unit 24) and at $360 \mathrm{mbsf}$ (top of Unit 31). These breaks correspond to the tops of massive flow units.

\section{MAGNETIC REMANENCE}

Magnetic remanence was measured on board ship using a $2 \mathrm{G}$ cryogenic magnetometer, in Oxford using either a CCL cryogenic magnetometer or a Molspin spinner magnetometer, and at BGR using a $2 \mathrm{G}$ cryogenic magnetometer. General procedure involved stepwise alternating field (AF) demagnetization up to fields of $100 \mathrm{mT}$. Stable remanence directions were defined using a least-squares algorithm (Kirschvink, 1980).

Above about $360 \mathrm{mbsf}$, the majority of samples show a stable single component on $\mathrm{AF}$ demagnetization, with a high degree of linearity on vector end-point diagrams and clustered directions in stereographic projection (Fig. $2 \mathrm{~A}$ ). They generally have some lowcoercivity magnetization, which is generally randomly oriented and can be removed by $10 \mathrm{mT}$ of $\mathrm{AF}$ treatment. This contrasts with other drilling-induced remanences (e.g., Allerton et al., 1995), which typically have consistent, steep inclinations. In this upper part of the hole, the inclinations of the high-coercivity component are generally close to horizontal (mean inclination $=-7.5^{\circ} \pm 10.2^{\circ}$, compared with an expected paleoinclination $=-7^{\circ}$ ).

In this upper part of the hole there is a suggestion that the inclinations vary in a systematic, cyclical manner, with an amplitude of about $10^{\circ}$ and a wavelength of about $70 \mathrm{~m}$ (Fig. 3A, B). This behavior may reflect secular variation of the sequence, suggesting that eruption was relatively continuous, at a rate of about $150 \mathrm{~m}$ in a few thousand years. Some individual samples have anomalous inclinations (Samples 148-896A-7R-1, 12-14 cm; 8R-1, 29-31 cm; 8R-1, 95-97 $\mathrm{cm}$; and $12 \mathrm{R}-2,23-25 \mathrm{~cm}$ ), and yet all the other characteristics of the magnetization are similar to the rest of the sequence. It is most likely that these are from pillow lavas that were moved after they had acquired their magnetization.

Below 360 mbsf, the AF demagnetization produces a less clearly linear magnetization, shown by a large maximum angular deviation (MAD) of $4^{\circ}$, compared to a MAD of $2^{\circ}$ in the upper section. On a stereographic projection, the majority of samples from below 360 mbsf plot as a segment of a great circle or as highly scattered paths, which indicates the presence of multiple components with overlapping coercivity spectra (Fig. 2B). In this part of the hole, inclinations are very scattered even from the same lithologic unit (cf., e.g., results from Unit 37; Table 1). We do not consider that the directions from this lower interval represent any consistently meaningful measurement of the paleomagnetic field.

Samples from massive flow Units $31-36$ and 49 and pillow Unit 47 commonly carry a high-coercivity component that is not removed during AF demagnetization. Hematite reported in some thin sections from the massive Units 31-36 may be the carrier of this high-coercivity magnetization.

Although we cannot compare full directional data (declination and inclination) from the core as a whole, it is possible to compare directional data from individual core pieces for the few cases where more than one sample for each piece of core has been measured. Of the 37 pairs of samples, 22 are separated by $10^{\circ}$ or less, and 28 are separated by $20^{\circ}$ or less. This compares with the stable inclinations for the same samples from the same pieces, for which 36 pairs are separated by less than $10^{\circ}$ of inclination, and one pair is separated by $33^{\circ}$ of inclination. The significantly larger scatter for the full directional data at least partly results from the inclusion of pieces with multiple subpieces - that is, broken pieces of core that can be fitted together. The larger values $\left(>21^{\circ}\right)$ are all from such pieces, except for Samples 148-896A-17R-2, 28-30 cm, and 17R-2, 38-40 cm, which suggests that these pieces were poorly matched before the cores were sliced. Unfortunately, in the section below $360 \mathrm{mbsf}$, where the fidelity of the magnetic signal is in doubt, only two pieces with two samples per piece were sampled. One of these pairs gives an inclination difference of $33^{\circ}$; the other pair gives an inclination difference of $3^{\circ}$.

The absence of the drilling-induced remanence so often reported for ODP cores (e.g., Ade-Hall and Johnson, 1976; Johnson, 1978; Lowrie and Kent, 1978) makes it possible to use the initial intensity of the sample $\left(J_{O}\right)$ as a direct measure of the in situ remanence. There is no need to apply a correction such as those described for other studies of the magnetism of the ocean basement (e.g., Pariso and Johnson, 1991). Plotting the intensity against depth below basement (Fig. 3C) shows a small change below $330 \mathrm{mbsf}$ and a more significant change beneath 360 mbsf (see Table 2 ).

The stability of magnetic remanence is indicated by the median destructive field (MDF, the AF required to demagnetize the sample to one-half its initial intensity). Plotted against depth (Fig. 3D), this property also shows a distinct reduction below $330 \mathrm{mbsf}$ and no significant change below 360 mbsf (see Table 2).

\section{INDUCED MAGNETIZATION}

The magnetic susceptibility of the samples is dependent on the composition, concentration, and grain size of the magnetic minerals in the rock. Although paramagnetic minerals, such as amphiboles and pyroxenes, make some contribution, the susceptibility in these rocks is dominated by the effects of ferro- and ferrimagnetic minerals. The 
Table 1. Paleomagnetic and rock magnetic measurements of samples from Hole 896A.

\begin{tabular}{|c|c|c|c|c|c|c|c|c|c|c|c|}
\hline $\begin{array}{l}\text { Core, section, } \\
\text { interval }(\mathrm{cm})\end{array}$ & Piece & Lithology & Unit & $\begin{array}{l}\text { Depth } \\
\text { (mbsf) }\end{array}$ & $\begin{array}{c}\text { Bulk } \\
\text { susceptibility } \\
\left(\times 10^{-3}\right. \\
\text { SI units })\end{array}$ & $\begin{array}{c}J_{0} \\
(\mathrm{~A} / \mathrm{m})\end{array}$ & $\begin{array}{c}\text { Relative } \\
\text { declination } \\
\left({ }^{\circ}\right)\end{array}$ & $\begin{array}{l}\text { Inclination } \\
\left({ }^{\circ}\right)\end{array}$ & $\begin{array}{c}\text { Maximum } \\
\text { angular } \\
\text { deviation } \\
\left({ }^{\circ}\right)\end{array}$ & $\begin{array}{l}\text { Median } \\
\text { destructive } \\
\text { field } \\
(\mathrm{mT})\end{array}$ & $\begin{array}{c}J_{r s} \\
(\mathrm{~A} / \mathrm{m})\end{array}$ \\
\hline $148-896 \mathrm{~A}=$ & & & & & & & & & & & \\
\hline IR-1, 47 & II & $\mathrm{P}$ & 3 & 195.6 & 8.6 & 12.4 & 299 & -2 & & 50 & \\
\hline IR-1.87 & 18 & P & 3 & 196.0 & 12.5 & 16.2 & 268 & -12 & & 28 & 220.1 \\
\hline $2 \mathrm{R}-\mathrm{I}, 3$ & 1 & M & 4 & 201.1 & 14.3 & 7.0 & 69 & & 1.0 & 10 & \\
\hline $2 \mathrm{R}-1.82$ & 19 & M & 7 & 201.7 & 13.4 & & & & & 15 & 178.8 \\
\hline $3 R-1.19$ & 4 & $\mathrm{P}$ & 8 & 210.1 & 2.0 & 4.8 & 90 & -19 & 2.0 & 37 & \\
\hline $3 \mathrm{R}-1,38$ & 7 & $\mathrm{P}$ & 8 & 210.3 & 3.7 & 10.3 & 67 & -10 & & & \\
\hline $3 \mathrm{R}-1,45$ & 7 & $\mathrm{P}$ & 8 & 210.4 & 4.1 & 9.6 & 67 & -8 & 2.0 & 25 & \\
\hline $3 \mathrm{R}-1,63$ & 8 & P & 8 & 210.5 & 4.6 & 11.5 & 289 & -7 & 4.0 & 27 & \\
\hline $3 R-1,68$ & 8 & $\mathrm{P}$ & 8 & 210.6 & 9.4 & 9.3 & 284 & -7 & 1.0 & 17 & \\
\hline $3 \mathrm{R}-1,78$ & 9 & $M$ & 9 & 210.7 & 10.2 & 8.6 & 118 & -9 & & 18 & 203.5 \\
\hline $3 R-1,96$ & 9 & M & 9 & 210.9 & 12.5 & & 282 & -5 & 1.0 & & \\
\hline $4 \mathrm{R}-1.44$ & $9 \mathrm{a}$ & $\mathrm{P}$ & 9 & 219.3 & 12.1 & 11.5 & 110 & -8 & & 24 & 206.3 \\
\hline $4 \mathrm{R}-1,49$ & $9 \mathrm{a}$ & $P$ & 9 & 219.4 & 10.0 & 7.6 & 105 & -12 & & & \\
\hline $4 R-1.68$ & $9 \mathrm{c}$ & $\mathrm{P}$ & 9 & 219.6 & 13.2 & 20.7 & 139 & -8 & & 25 & \\
\hline $5 \mathrm{R}-2,27$ & lc & $\mathrm{P}$ & 10 & 230.2 & 7.4 & 13.1 & 192 & -13 & 1.6 & 26 & \\
\hline $5 R-2,38$ & Id & $\mathrm{P}$ & 10 & 229.7 & 3.2 & 8.3 & 233 & -14 & & 50 & \\
\hline $5 R-2,125$ & 13 & P & 10 & 231.2 & 6.6 & 11.5 & 276 & -4 & 2.9 & 26 & \\
\hline $5 R-2,126$ & 13 & $\mathrm{P}$ & 10 & 230.6 & 5.2 & & 280 & -3 & 3.0 & & \\
\hline $5 \mathrm{R}-3,43$ & $5 b$ & M & 11 & 231.2 & 10.9 & 3.7 & 334 & -13 & & 17 & 189.9 \\
\hline $5 \mathrm{R}-3,62$ & 8 & M & 11 & 231.4 & 11.7 & 15.2 & 235 & -9 & & 15 & 292.2 \\
\hline $6 \mathrm{R}-1,39$ & $5 a$ & $\mathrm{P}$ & 12 & 238.3 & 6.6 & 80.2 & 92 & -13 & 3.0 & . & \\
\hline $6 \mathrm{R}-1,48$ & $5 b$ & $\mathrm{P}$ & 12 & 238.4 & 7.9 & 13.2 & 75 & -5 & 1.2 & 26 & \\
\hline $6 \mathrm{R}-1,52$ & $5 c$ & P & 12 & 238.4 & 6.1 & 16.0 & 83 & -15 & & 62 & \\
\hline $6 \mathrm{R}-1,93$ & $9 \mathrm{~b}$ & $\mathrm{P}$ & 12 & 238.8 & 17.4 & 22.5 & 344 & -19 & 1.0 & 15 & \\
\hline $6 \mathrm{R}-2,3$ & la & $\mathrm{P}$ & 12 & 239.4 & 1.4 & 3.1 & 56 & -17 & & 60 & 51.2 \\
\hline $6 \mathrm{R}-2,11$ & $1 \mathrm{~b}$ & $\mathrm{P}$ & 12 & 239.5 & 0.9 & 1.9 & 279 & -15 & 3.0 & 44 & \\
\hline $6 \mathrm{R}-2,89$ & $10 \mathrm{a}$ & $\mathrm{P}$ & 12 & 240.3 & 4.5 & 12.3 & 43 & -10 & & 40 & \\
\hline $6 \mathrm{R}-3,8$ & 1 & $\mathrm{P}$ & 12 & 240.9 & 7.4 & 15.9 & 255 & -16 & & 30 & 245.5 \\
\hline $7 R-1,12$ & 3 & $\mathrm{P}$ & 13 & 247.5 & 1.2 & 1.0 & 35 & 31 & 1.3 & 30 & \\
\hline $7 R-1,38$ & 7 & $\mathrm{P}$ & 13 & 247.8 & 2.2 & 3.5 & 312 & 0 & 2.0 & 40 & \\
\hline $7 \mathrm{R}-1,58$ & 10 & P & 13 & 248.0 & 5.0 & 12.0 & 222 & -12 & & 34 & 228.6 \\
\hline $7 \mathrm{R}-1.78$ & 13 & $\mathrm{P}$ & 14 & 248.2 & 6.8 & 12.4 & 87 & -9 & 0.0 & & \\
\hline $7 \mathrm{R}-1.80$ & 13 & $P$ & 14 & 248.2 & 10.3 & 15.2 & 88 & -10 & & 17 & 268.7 \\
\hline $7 \mathrm{R}-1,101$ & 15 & $\mathrm{P}$ & 14 & 248.4 & 6.8 & 12.7 & 215 & -11 & & & \\
\hline $8 \mathrm{R}-1,29$ & 7 & $\mathrm{P}$ & 14 & 257.4 & 9.1 & 11.4 & 183 & -34 & & & 251.2 \\
\hline $8 \mathrm{R}-1,95$ & 19 & $\mathrm{P}$ & 14 & 258.1 & 4.5 & 8.8 & 39 & -60 & 3.8 & 28 & \\
\hline $9 \mathrm{R}-1,32$ & 6 & $\mathrm{P}$ & 14 & 267.0 & 11.8 & 9.7 & 86 & 19 & 1.2 & & \\
\hline $9 \mathrm{R}-1,42$ & 7 & $\mathrm{P}$ & 14 & 267.1 & 5.2 & 11.1 & 251 & -1 & 2.0 & 28 & \\
\hline $9 \mathrm{R}-1,135$ & 25 & $\mathrm{P}$ & 14 & 268.1 & 7.4 & 7.3 & 72 & -11 & 2.0 & 18 & \\
\hline $9 \mathrm{R}-1,138$ & 25 & $\mathrm{P}$ & 14 & 268.1 & 11.9 & 10.9 & 71 & -6 & & 20 & 244.0 \\
\hline $9 \mathrm{R}-2,4$ & 1 & $\mathrm{P}$ & 14 & 268.2 & 5.0 & 14.8 & 211 & -7 & & 45 & 272.3 \\
\hline $9 \mathrm{R}-2,6$ & 1 & $P$ & 14 & 268.2 & 5.6 & 10.6 & 210 & -7 & 1.0 & 28 & \\
\hline $9 \mathrm{R}-2,44$ & 4 & $\mathrm{P}$ & 14 & 268.5 & 7.2 & 10.8 & 236 & -3 & 3.0 & 23 & \\
\hline $10 \mathrm{R}-1,70$ & $8 b$ & M & 14 & 277.1 & 5.6 & 12.4 & 220 & -3 & & 25 & \\
\hline $10 \mathrm{R}-1.80$ & $8 d$ & M & 14 & 277.2 & 10.4 & 12.3 & 33 & -11 & & & \\
\hline $10 \mathrm{R}-1.85$ & $8 \mathrm{e}$ & M & 14 & 277.3 & 9.7 & 15.6 & 51 & -2 & & 27 & 265.0 \\
\hline $10 R-1,104$ & $9 b$ & M & 14 & 277.4 & 6.8 & 16.6 & 90 & -1 & 2.0 & 19 & \\
\hline $10 R-1.116$ & $9 \mathrm{c}$ & M & 14 & 277.6 & 8.1 & 15.3 & 16 & -1 & & 34 & 265.8 \\
\hline IIR-1, 32 & $3 d$ & $\mathrm{P}$ & 14 & 286.3 & 11.2 & 9.8 & 19 & -5 & & 17 & 216.5 \\
\hline $11 \mathrm{R}-1.37$ & $3 \mathrm{e}$ & $\mathrm{P}$ & 14 & 286.4 & 10.3 & 7.5 & 16 & -3 & & & \\
\hline $\mid 1 \mathrm{R}-1.51$ & $3 e$ & P & 14 & 286.5 & 13.5 & 10.7 & 13 & -9 & 0.3 & 13 & \\
\hline $11 R-1,120$ & $10 \mathrm{c}$ & $\mathrm{P}$ & 14 & 287.2 & 5.6 & 9.2 & 126 & -12 & 3.0 & 34 & \\
\hline IIR-3, 19 & 4 & M & 15 & 289.1 & 13.4 & 16.0 & 56 & -7 & 1.0 & 20 & \\
\hline $11 \mathrm{R}-3,90$ & 15 & P & 14 & 289.9 & 11.8 & 18.7 & 305 & -8 & 1.7 & 17 & \\
\hline $12 \mathrm{R}-1,20$ & $3 b$ & $\mathrm{P}$ & 16 & 295.3 & 11.7 & 10.2 & 41 & -13 & & & \\
\hline $12 \mathrm{R}-1,53$ & 8 & M & 16 & 296.1 & 14.4 & 11.3 & 337 & -12 & 0.8 & & \\
\hline $12 \mathrm{R}-1.63$ & $9 \mathrm{c}$ & M & 16 & 295.7 & 13.4 & & 223 & -8 & 1.0 & & \\
\hline $12 \mathrm{R}-1,68$ & $9 \mathrm{c}$ & M & 16 & 296.3 & 13.6 & 15.3 & 222 & -8 & & 15 & 264.2 \\
\hline $12 \mathrm{R}-1,78$ & $10 b$ & $\mathrm{M}$ & 16 & 296.4 & 13.5 & 13.6 & 110 & -12 & & 16 & 249.2 \\
\hline $12 \mathrm{R}-1.80$ & $10 \mathrm{~b}$ & M & 16 & 296.4 & 12.8 & 13.6 & 102 & -21 & & 16 & \\
\hline $12 \mathrm{R}-1,82$ & $10 b$ & M & 16 & 295.9 & 11.1 & 9.7 & 104 & -16 & 2.0 & 15 & \\
\hline $12 \mathrm{R}-\mathrm{I}, 141$ & $18 \mathrm{~b}$ & M & 16 & 296.5 & 9.4 & 5.8 & 295 & -11 & 2.0 & 20 & \\
\hline $12 \mathrm{R}-2,9$ & Ib & M & 17 & 297.1 & 17.8 & & 262 & -12 & & & 286.4 \\
\hline $12 \mathrm{R}-2,23$ & 2 & M & 17 & 297.2 & 13.8 & 8.6 & 42 & 17 & 1.0 & & \\
\hline $14 \mathrm{R}-2,28$ & 3 & $\mathrm{P}$ & 19 & 316.3 & 6.7 & 14.1 & 25 & -14 & & 31 & 239.6 \\
\hline $14 \mathrm{R}-2,113$ & $16 \mathrm{a}$ & $\dot{M}$ & 21 & 317.1 & 17.0 & 11.9 & 91 & -8 & & 13 & 216.7 \\
\hline $14 \mathrm{R}-2,118$ & $16 b$ & M & 21 & 317.2 & 16.1 & 6.2 & 93 & -4 & 2.0 & & \\
\hline $14 \mathrm{R}-3,16$ & 3 & M & 21 & 317.7 & 8.1 & 10.8 & 56 & -6 & & & \\
\hline $14 \mathrm{R}-3.65$ & 11 & M & 21 & 318.2 & 10.4 & & 14 & -11 & 1.0 & & \\
\hline $15 R-1,3$ & 1 & M & 22 & 324.3 & 0.0 & 10.6 & 326 & -21 & 1.0 & 12 & \\
\hline $15 \mathrm{R}-1.103$ & 15 & $\mathrm{P}$ & 23 & 325.3 & 8.9 & 16.2 & 24 & -12 & & 33 & 294.5 \\
\hline $15 \mathrm{R}-1,105$ & 15 & $\mathrm{P}$ & 23 & 325.4 & 7.6 & 11.1 & 13 & -10 & 1.0 & & \\
\hline $15 R-1,128$ & $18 \mathrm{a}$ & $\mathrm{P}$ & 23 & 325.6 & 7.8 & 13.3 & 168 & -13 & 1.3 & 28 & \\
\hline $15 R-2,73$ & $7 b$ & $\mathrm{P}$ & 23 & 326.4 & 12.9 & 9.8 & 72 & -9 & 1.0 & 16 & \\
\hline $15 R-2,123$ & 16 & $\mathrm{P}$ & 23 & 326.9 & 9.7 & 5.8 & 150 & -9 & 2.0 & 21 & \\
\hline $16 \mathrm{R}-1.21$ & $3 b$ & $\mathrm{P}$ & 24 & 334.1 & 16.9 & 13.2 & 54 & -6 & & 17 & \\
\hline $16 \mathrm{R}-1,32$ & $3 b$ & $\mathrm{P}$ & 24 & 334.2 & 18.1 & 9.4 & 56 & -8 & & 17 & 253 \\
\hline $16 \mathrm{R}-1,66$ & 8 & M & 24 & 334.6 & 22.0 & 11.2 & 69 & -10 & & & \\
\hline $16 \mathrm{R}-1,124$ & $9 \mathrm{~d}$ & M & 24 & 335.1 & 19.6 & 3.2 & 230 & -5 & 2.0 & 9 & \\
\hline $16 \mathrm{R}-2,11$ & la & M & 24 & 335.3 & 24.5 & 4.3 & 334 & -8 & & & \\
\hline $16 \mathrm{R}-2,14$ & $\mathrm{Ib}$ & M & 24 & 335.3 & 23.2 & 4.3 & 326 & -2 & 0.0 & 7 & \\
\hline $16 R-2,27$ & lc & M & 24 & 335.7 & 24.2 & 4.4 & 330 & -6 & 0.6 & 5 & \\
\hline 16R-2, 34 & Ic & M & 24 & 335.6 & 34.4 & 5.0 & 339 & -6 & & 8 & 179.3 \\
\hline $16 \mathrm{R}-2,50$ & le & M & 24 & 335.7 & 26.6 & 5.8 & 216 & 14 & 2.0 & 9 & \\
\hline $16 \mathrm{R}-2,55$ & le & M & 24 & 335.8 & 21.1 & 2.4 & 217 & 10 & 2.0 & 13 & \\
\hline
\end{tabular}


Table 1 (continued).

\begin{tabular}{|c|c|c|c|c|c|c|c|c|c|c|c|c|}
\hline $\begin{array}{l}\text { Core, section, } \\
\text { interval }(\mathrm{cm})\end{array}$ & Piece & Lithology & Unit & $\begin{array}{l}\text { Depth } \\
\text { (mbsf) }\end{array}$ & $\begin{array}{c}\text { Bulk } \\
\text { susceptibility } \\
\left(\times 10^{-3}\right. \\
\text { SI units) }\end{array}$ & $\begin{array}{c}J_{0} \\
(\mathrm{~A} / \mathrm{m})\end{array}$ & $\begin{array}{c}\text { Relative } \\
\text { declination } \\
\left({ }^{\circ}\right)\end{array}$ & $\begin{array}{c}\text { Inclination } \\
\left({ }^{\circ}\right)\end{array}$ & $\begin{array}{c}\text { Maximum } \\
\text { angular } \\
\text { deviation } \\
\left({ }^{\circ}\right)\end{array}$ & $\begin{array}{c}\text { Median } \\
\text { destructive } \\
\text { field } \\
(\mathrm{mT})\end{array}$ & $\begin{array}{c}J_{r \mathrm{~s}} \\
(\mathrm{~A} / \mathrm{m})\end{array}$ & $\begin{array}{l}H_{\text {sat }} \\
\text { (T) }\end{array}$ \\
\hline \multicolumn{13}{|l|}{ 148-896A- } \\
\hline $16 \mathrm{R}-2,113$ & $4 b$ & M & 24 & 336.3 & 17.3 & 6.6 & 276 & -5 & 2.0 & 7 & & \\
\hline $16 \mathrm{R}-2,130$ & $4 c$ & $\mathrm{M}$ & 24 & 336.6 & 20.4 & 7.9 & 284 & -14 & & 10 & & \\
\hline $16 \mathrm{R}-2.132$ & $4 c$ & M & 24 & 336.6 & 19.3 & 7.4 & 279 & -6 & & 10 & 223.3 & 0.58 \\
\hline $16 \mathrm{R}-3,55$ & Id & M & 24 & 337.3 & 18.1 & 2.0 & 279 & -13 & 2.0 & 8 & & \\
\hline $16 \mathrm{R}-3,81$ & $4 a$ & M & 24 & 337.5 & 19.6 & 4.5 & 90 & -9 & 3.0 & 8 & & \\
\hline $16 \mathrm{R}-3,85$ & $4 b$ & $\mathrm{M}$ & 24 & 337.5 & 0.0 & 4.2 & 110 & -12 & 2.1 & 7 & & \\
\hline $16 \mathrm{R}-3,88$ & $4 b$ & M & 24 & 337.6 & 23.0 & 4.4 & 111 & -3 & & 9 & 211.0 & 0.25 \\
\hline $17 \mathrm{R}-1.1$ & la & $\mathrm{P}$ & 25 & 343.5 & 19.3 & 14.4 & 344 & 15 & & 17 & 273.0 & 0.09 \\
\hline $17 \mathrm{R}-1,82$ & $10 \mathrm{~b}$ & $\mathrm{P}$ & 26 & 344.3 & 20.2 & 4.8 & 206 & -10 & & 16 & 213.7 & 0.10 \\
\hline $17 \mathrm{R}-1.85$ & $10 \mathrm{~b}$ & $\mathrm{P}$ & 26 & 344.4 & 16.7 & 3.7 & 204 & -2 & & & & \\
\hline $17 \mathrm{R}-1,136$ & $16 a$ & $\mathrm{P}$ & 27 & 344.9 & 23.5 & 6.6 & 285 & -8 & 0.8 & 10 & & \\
\hline $17 R-1.139$ & $16 \mathrm{~b}$ & $\mathrm{P}$ & 27 & 344.9 & 22.2 & 7.2 & 280 & -7 & 1.0 & 9 & & \\
\hline $17 \mathrm{R}-2.19$ & $3 a$ & $\mathrm{P}$ & 27 & 345.2 & 21.1 & 8.1 & 148 & -10 & 0.9 & 13 & & \\
\hline $17 \mathrm{R}-2,40$ & $3 \mathrm{e}$ & $\mathrm{P}$ & 27 & 345.4 & 24.2 & 4.7 & 317 & -6 & 2.0 & 10 & & \\
\hline $17 R-2,126$ & 11 & $\mathrm{P}$ & 27 & 346.3 & 23.5 & 16.8 & 44 & -6 & & 13 & 287.1 & 0.16 \\
\hline $17 R-2,140$ & $12 b$ & $\mathrm{P}$ & 27 & 346.4 & 22.2 & 7.3 & 193 & 0 & 1.0 & 9 & & \\
\hline $17 \mathrm{R}-3,37$ & $4 a$ & $\mathrm{P}$ & 27 & 346.8 & 22.5 & 15.6 & 65 & -3 & 2.0 & 13 & & \\
\hline $17 \mathrm{R}-3,57$ & $4 a$ & $\mathrm{P}$ & 27 & 347.0 & 21.8 & 9.6 & 57 & -9 & 2.0 & 4 & 320.4 & 0.08 \\
\hline $17 \mathrm{R}-3,86$ & 8 & $\mathrm{P}$ & 28 & 347.3 & 16.9 & 10.2 & 215 & -6 & 2.0 & 10 & . & \\
\hline $17 R-3,89$ & 8 & $\mathrm{P}$ & 28 & 347.3 & 22.4 & 9.7 & 217 & -7 & & 14 & 233.9 & 0.08 \\
\hline $17 \mathrm{R}-4,28$ & $2 \mathrm{~d}$ & P & 28 & 348.2 & 23.5 & 12.1 & 239 & -2 & & 12 & 240.6 & 0.20 \\
\hline $17 R-4,36$ & $2 d$ & $\mathrm{P}$ & 28 & 348.3 & 22.7 & 8.1 & 275 & -2 & 1.0 & 9 & & \\
\hline $17 \mathrm{R}-4,97$ & 9 & $\dot{M}$ & 28 & 348.9 & 27.3 & 8.4 & 149 & 3 & & & & \\
\hline $17 R-4,99$ & 9 & M & 28 & 348.9 & 30.3 & 15.5 & 149 & 2 & & 8 & & \\
\hline $17 \mathrm{R}-5,21$ & 4 & M & 28 & 349.6 & 23.8 & 1.7 & 133 & -7 & 0.5 & 10 & & \\
\hline $18 \mathrm{R}-1.72$ & $6 b$ & $\mathrm{P}$ & 29 & 353.8 & 20.1 & 13.5 & 230 & 11 & & 17 & & \\
\hline $18 \mathrm{R}-1.102$ & $9 b$ & $\mathrm{P}$ & 29 & 354.1 & 21.0 & 13.8 & 166 & 0 & & 17 & 275.9 & 0.08 \\
\hline $18 \mathrm{R}-1,127$ & IIb & $\mathrm{P}$ & 29 & 354.4 & 24.4 & 6.2 & 250 & 14 & 1.3 & 18 & & \\
\hline $18 R-2.60$ & 10 & $\mathrm{P}$ & 30 & 355.2 & 8.8 & 8.3 & 322 & -28 & & 24 & & \\
\hline $19 \mathrm{R}-1.23$ & $5 a$ & $\mathrm{P}$ & 30 & 356.2 & 25.8 & 13.6 & 115 & -26 & & 19 & 456.4 & 0.95 \\
\hline $20 \mathrm{R}-1.63$ & 15 & M & 31 & 364.1 & 36.5 & 10.4 & 267 & -52 & & 18 & & \\
\hline $20 \mathrm{R}-1,108$ & 22 & M & 31 & 364.5 & 47.0 & 4.4 & 288 & -6 & & 9 & & \\
\hline $21 \mathrm{R}-1.58$ & 10 & M & 31 & 373.6 & 30.0 & 1.4 & 338 & -16 & & 17 & & \\
\hline $21 R-1.105$ & 17 & $M$ & 31 & 374.1 & 20.1 & 1.4 & 355 & -11 & & & & \\
\hline $21 \mathrm{R}-1.137$ & 20 & M & 31 & 374.4 & 19.6 & 1.0 & 130 & -5 & 2.0 & 8 & 202.5 & 0.05 \\
\hline $21 \mathrm{R}-2.10$ & 2 & M & 31 & 376.1 & 20.1 & 1.4 & 137 & -6 & & & & \\
\hline 2 IR- 2.85 & $9 \mathrm{~b}$ & M & 31 & 375.3 & 47.0 & 2.4 & 139 & 13 & & 3 & & \\
\hline $21 \mathrm{R}-2,118$ & $11 \mathrm{~b}$ & M & 32 & 375.7 & 23.5 & 6.7 & 52 & -35 & 0.6 & 9 & & \\
\hline $21 R-2,133$ & $13 \mathrm{a}$ & $\mathrm{M}$ & 32 & 375.8 & 31.0 & 3.8 & 58 & -59 & & 10 & & \\
\hline $22 \mathrm{R}-1,45$ & $5 b$ & M & 32 & 383.1 & 20.8 & 6.6 & 184 & -37 & 1.0 & 14 & & \\
\hline $22 \mathrm{R}-\mathrm{I} .107$ & 13 & M & 32 & 383.7 & 36.1 & 13.0 & 233 & -31 & & 21 & & \\
\hline $22 \mathrm{R}-3,11$ & 2 & M & 33 & 385.7 & 29.5 & 3.8 & 116 & -37 & & 9 & & \\
\hline $22 \mathrm{R}-3,26$ & 3 & M & 33 & 385.9 & 50.7 & 5.0 & 84 & -44 & & 10 & & \\
\hline $22 \mathrm{R}-4,9$ & $\mathrm{Ib}$ & M & 33 & 388.0 & 25.2 & 4.5 & 284 & -38 & 1.3 & 11 & & \\
\hline $23 \mathrm{R}-1,18$ & 2 & B & 35 & 392.3 & 36.8 & 1.0 & 185 & 52 & & 4 & & \\
\hline $23 \mathrm{R}-1.77$ & 11 & $M$ & 36 & 392.9 & 26.5 & 4.6 & 67 & -7 & & 18 & & \\
\hline $23 R-2.20$ & $2 \mathrm{a}$ & M & 36 & 393.8 & 19.6 & 1.4 & 97 & -12 & 2.0 & 8 & & \\
\hline $23 \mathrm{R}-2.48$ & 4 & M & 36 & 394.1 & 22.0 & 1.3 & 75 & -7 & 3.0 & 7 & & \\
\hline $23 \mathrm{R}-2,82$ & 7 & M & 36 & 394,4 & 18.6 & 0.8 & 173 & -20 & 3.0 & 10 & 197.5 & 0.05 \\
\hline $23 R-3,6$ & 1 & M & 36 & 395.1 & 37.7 & 2.5 & 43 & 43 & & 5 & & \\
\hline $23 \mathrm{R}-3,132$ & 17 & $\mathrm{M}$ & 36 & 396.3 & 40.1 & 3.3 & 187 & 48 & & 5 & 159.2 & 0.13 \\
\hline $24 R-1,16$ & 1 & M & 36 & 402.0 & 20.2 & 2.3 & 224 & 66 & 6.4 & 3 & & \\
\hline $24 \mathrm{R}-1,61$ & 4 & M & 36 & 402.4 & 23.4 & 0.5 & 350 & 0 & 7.0 & 8 & & \\
\hline $24 \mathrm{R}-\mathrm{I} .122$ & 8 & M & 36 & 403.0 & 18.7 & 2.4 & 158 & 55 & 6.3 & 5 & & \\
\hline $24 \mathrm{R}-\mathrm{I}, 125$ & 8 & M & 36 & 403.1 & 45.3 & 4.1 & 172 & 22 & & 5 & & \\
\hline $24 \mathrm{R}-2,85$ & 10 & $M$ & 36 & 404.2 & 23.0 & 1.2 & 272 & 44 & 3.0 & 13 & & \\
\hline $24 R-2.114$ & 13 & M & 36 & 404.4 & 24.2 & 1.3 & 345 & 27 & 3.0 & 8 & 195.6 & 0.05 \\
\hline $24 \mathrm{R}-3,52$ & 5 & M & 36 & 405.3 & 23.3 & 0.5 & 144 & 32 & 4.7 & 6 & & \\
\hline $24 R-3,140$ & $14 \mathrm{~b}$ & M & 36 & 406.2 & 44.7 & 2.9 & 214 & 41 & & 2 & & \\
\hline $24 \mathrm{R}-4.53$ & 7 & $M$ & 36 & 406.7 & 24.3 & 1.3 & 242 & 24 & 2.0 & 7 & & \\
\hline $24 R-5,90$ & 9 & M & 36 & 408.6 & 25.1 & 1.6 & 35 & -30 & 3.0 & 4 & 171.2 & 0.05 \\
\hline $24 \mathrm{R}-5,118$ & 12 & $\mathrm{M}$ & 36 & 375.7 & 23.5 & 1.7 & 154 & -36 & 5.6 & 5 & & \\
\hline $25 \mathrm{R}-1.34$ & 6 & $\mathrm{P}$ & 37 & 411.6 & 13.7 & 2.7 & 287 & 15 & 5.0 & 15 & 257.4 & 0.07 \\
\hline $25 \mathrm{R}-1.49$ & $6 b$ & $\mathrm{P}$ & 37 & 411.8 & 20.3 & 4.5 & 310 & 12 & & 20 & & \\
\hline $25 \mathrm{R}-1,141$ & 17 & P & 37 & 412.7 & 14.6 & 2.0 & 144 & 32 & 2.0 & 10 & & \\
\hline $25 R-2,89$ & 16 & P & 37 & 413.7 & 11.2 & 2.4 & 28 & -31 & 3.0 & 15 & 244.9 & 0.08 \\
\hline $25 \mathrm{R}-3.52$ & 7 & $\mathrm{P}$ & 37 & 414.7 & 19.2 & 3.6 & 335 & 2 & & 17 & & \\
\hline $26 \mathrm{R}-1.63$ & $9 b$ & $M$ & 38 & 421.6 & 16.6 & 3.4 & 95 & 10 & & 9 & & \\
\hline $26 \mathrm{R}-1.118$ & 17 & $\mathrm{P}$ & 40 & 423.7 & 23.2 & 2.1 & 7 & 24 & 3.3 & 5 & & \\
\hline $26 \mathrm{R}-2,27$ & 4 & $\mathrm{P}$ & 41 & 422.8 & 18.2 & 4.5 & 97 & 16 & & 18 & & \\
\hline $26 \mathrm{R}-3,19$ & Ic & P & 42 & 433.7 & 17.1 & 2.2 & 284 & 17 & 1.9 & 17 & & \\
\hline $27 \mathrm{R}-1,18$ & Ib & P & 42 & 430.7 & 13.8 & 1.8 & 200 & 53 & & & & \\
\hline $27 \mathrm{R}-1.69$ & 8 & P & 43 & 431.2 & 41.0 & 2.8 & 124 & -2 & & 9 & & \\
\hline $27 \mathrm{R}-3,43$ & 5 & $\mathrm{P}$ & 47 & 433.8 & 17.1 & 2.3 & 300 & -8 & 6.0 & 14 & 294.3 & 0.07 \\
\hline $28 \mathrm{R}-1,120$ & 18 & $\mathrm{P}$ & 47 & 441.2 & 14.9 & 2.4 & 136 & 20 & 2.2 & 21 & & \\
\hline $28 \mathrm{R}-2.15$ & Ib & P & 47 & 441.6 & 25.1 & 3.3 & 94 & 22 & & 19 & & \\
\hline $28 \mathrm{R}-2,39$ & $3 b$ & P & 47 & 441.8 & 29.0 & 8.2 & 273 & 19 & & 16 & & \\
\hline $29 \mathrm{R}-1,93$ & 13 & $\dot{M}$ & 49 & 450.6 & 63.2 & 4.4 & 266 & 69 & & 4 & & \\
\hline $29 R-2.7$ & Ic & $\mathrm{P}$ & 50 & 451.2 & 22.4 & 0.6 & 4 & -13 & 11.0 & 9 & & \\
\hline $30 \mathrm{R}-1.36$ & 6 & B & 50 & 459.7 & 24.5 & 3.7 & 226 & -41 & & 20 & & \\
\hline $30 \mathrm{R}-1.98$ & $12 \mathrm{~b}$ & $\mathrm{P}$ & 50 & 460.3 & 29.4 & 0.5 & 277 & -50 & 3.5 & 14 & & \\
\hline
\end{tabular}

Notes: Lithology: $\mathrm{P}=$ pillow, $\mathrm{M}=$ massive, and $\mathrm{B}=$ breccia. The declination is measured relative to the arbitrary cut face of the core. The dashed lines mark the intervals discussed in the text. 

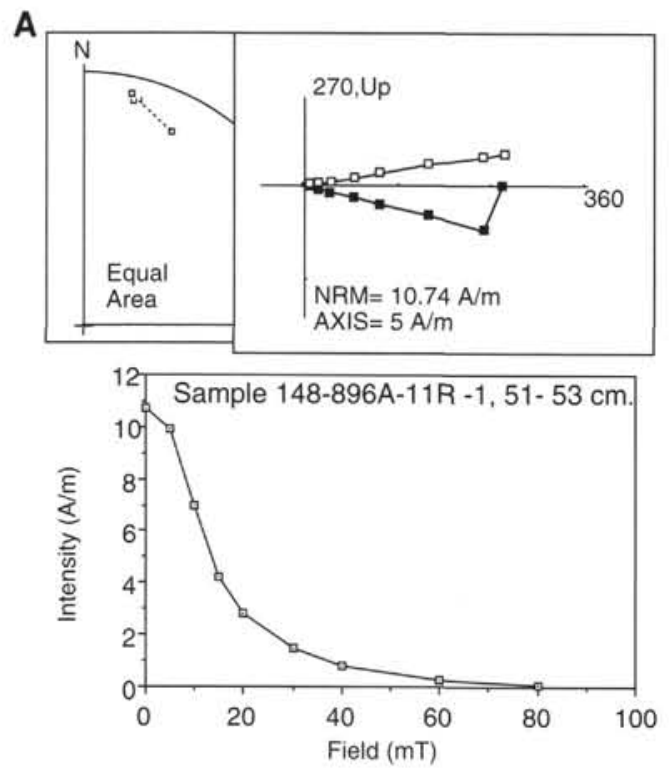

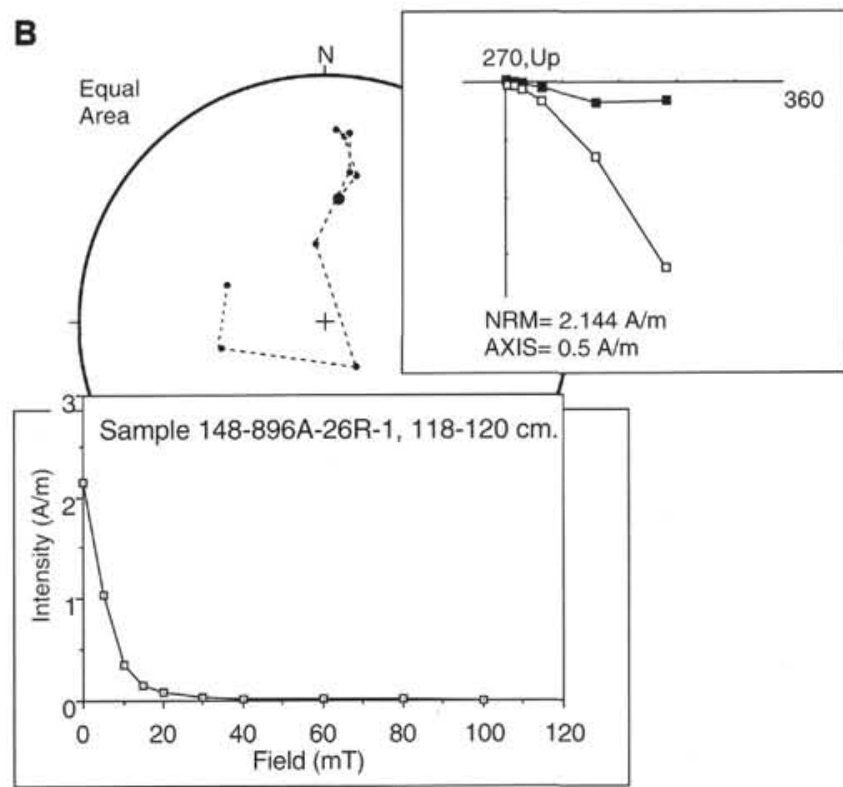

Figure 2. Alternating field demagnetization behavior. Orthogonal vector diagrams (solid squares are horizontal projections, open squares are vertical projections), stereographic projections, and intensity vs. demagnetizing field. A. Note the clear linearity during demagnetization and the tight grouping in stereographic projection. B. Sample 148-896A-26R-1, 118-120 cm. Note that the data plot as a segment of a great circle, and they do not plot as a linear segment on the orthogonal vector diagram.

bulk susceptibility was measured using a Geofyzika Brno Kappabridge, both aboard ship and in Oxford. The mean susceptibility is $17.6 \pm 10.6 \times 10^{-3} \mathrm{SI}$ units. The susceptibility increases below 330 mbsf, and again below $360 \mathrm{mbsf}$, with an increase in variability (Fig. 3E; Table 2).

\section{ISOTHERMAL REMANENT MAGNETIZATION}

The acquisition of isothermal remanent magnetization was investigated on board using an ASC impulse magnetizer. Further measurements were concluded in Oxford using a Molspin pulse magnetizer. The majority of samples exhibited a steep gradient of IRM acquisition up to the saturation level $\left(H_{\text {sal }}\right.$, defined as $95 \%$ of the maximum IRM intensity), after which no significant increase in intensity was noted with increasing applied field. Saturation of the samples in fields between 70 and $200 \mathrm{mT}$ indicates the predominance of (titano)magnetite and/or (titano)maghemite. The saturation of a few samples, mostly from massive flows, in fields greater than $200 \mathrm{mT}$ suggests the presence of additional, high-coercivity phases. Some anomalously high values (Samples 148-896A-16R-2, 132-134 cm, and $148-896 \mathrm{~A}-19 \mathrm{R}-1,23-25 \mathrm{~cm}$ ) may be associated with the occurrence of secondary hematite. There is some suggestion of a decrease in $H_{s a t}$ at about $350 \mathrm{mbsf}$. The saturation remanence, $J_{r s}$, has a mean value of $239 \pm 58 \mathrm{~A} / \mathrm{m}$.

\section{HIGH-TEMPERATURE SUSCEPTIBILITY}

The variation of magnetic susceptibility with temperature was studied to determine the mineralogy of the magnetic phases present in the samples. The procedure is similar to measurements of saturation remanence with temperature, but can give additional information about domain states. Low-field susceptibility was monitored during both heating and cooling cycles, up to a maximum temperature of $700^{\circ} \mathrm{C}$ using a CS2 attachment to a Geofyzika Brno Kappabridge. Powdered samples were measured in air, which may have enhanced the degree of oxidation in the measurements. This probably has the greatest effect on the cooling curves, after the samples had been heated to $700^{\circ} \mathrm{C}$. The system is particularly useful for distinguishing between titanomagnetites and titanomaghemites. Problems occur in the interpretation of phases with high Curie temperatures, as it is difficult to separate original phases from the products of alteration. Hematite, for example, indicated by susceptibilities between $600^{\circ}$ and $700^{\circ} \mathrm{C}$, may result either from in situ hydrothermal alteration or by breakdown of titanomaghemites. It is commonly difficult to identify Curie temperatures by taking the gradient of the slope, particularly for the first peak, because of the corruption of the slope introduced by the overlap of old phases and the formation of new phases makes the errors introduced by fitting a gradient unacceptable. Instead, we identified the peak temperature corresponding to each phase.

The temperature dependence of susceptibility of 23 samples was measured. On the basis of their high-temperature behavior, the samples can be separated into two different types.

Type I is irreversible, with the heating curve exhibiting either two peaks separated by a trough or two steep segments separated by a shallower segment. These curves are similar to saturation magnetization vs. temperature for extrusive rocks from Hole 504B (e.g., Pechersky et al., 1983; Furuta, 1983; Donovan and O'Reilly, 1983), which have been interpreted as titanomaghemites that invert to a multiphase product on heating. The thermomagnetic curves are reversible up to the first peak (typically close to $300^{\circ} \mathrm{C}$ ), but then exhibit irreversible behavior, and the characteristic "inversion" is in the range of $350^{\circ}-$ $470^{\circ} \mathrm{C}$ and peaks between $400^{\circ}$ and $520^{\circ} \mathrm{C}$ (see Table 3 ).

The cooling curves for the type I irreversible curves yield information on the complex products of inversion and subsequent oxidation. In some cases, the cooling curve has a gradual slope that peaks at about $200^{\circ} \mathrm{C}$ (type la; Fig. 4A, B). These probably represent inversion of the titanomaghemite to a titanomagnetite with a similar $\mathrm{Fe} / \mathrm{Ti}$ ratio. These curves are reversible on further heating and cooling. Some curves have a high-temperature peak (between $500^{\circ}$ and $600^{\circ} \mathrm{C}$ ) on cooling (type Ib; Fig. $4 \mathrm{C}$ ), which may correspond to a magnetite or Ti-depleted titanomagnetite resulting from exsolution during inversion. The third type of irreversible curve involves a hightemperature phase $\left(600^{\circ}-700^{\circ} \mathrm{C}\right)$ on cooling (type Ic, Fig. 4D), which 

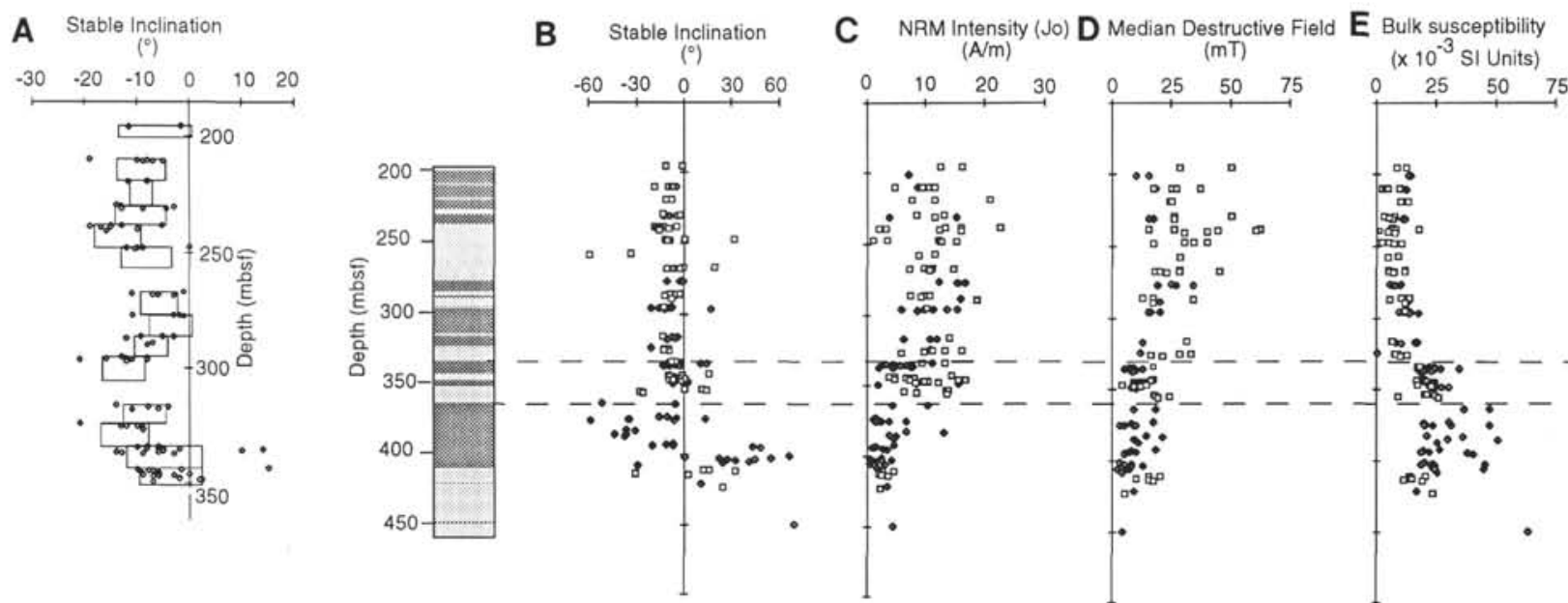

Figure 3. A-E. Magnetic properties vs. depth. Samples from massive flows are displayed as diamonds, and samples from pillow flows are squares. The lithostratigraphy is also marked schematically: light shaded areas are pillow flows and dark shaded areas are massive flows. In the inclination of the upper part of the hole (Fig. 3A), the boxes represent mean inclinations for individual cores and the depth over which the core was taken. Samples with an inclination that deviates significantly from the main group, as discussed in the text, have been omitted.

Table 2. Mean paleomagnetic and rock magnetic measurements from Hole 896A and upper part of Hole 504B (from the top of basement to 830 mbsf).

\begin{tabular}{|c|c|c|c|c|c|c|}
\hline Depth (mbsf) & Lithology & $\begin{array}{c}\text { Bulk } \\
\text { suceptibility } \\
\left(\times 10^{-3}\right. \\
\text { SI units })\end{array}$ & $\begin{array}{c}\text { NRM } \\
\text { intensity } \\
\left(J_{0}\right) \\
(\mathrm{A} / \mathrm{m})\end{array}$ & $\begin{array}{c}\text { Stable } \\
\text { inclination } \\
\left({ }^{\circ}\right)\end{array}$ & $\begin{array}{l}\text { Median } \\
\text { destructive } \\
\text { field } \\
\text { (mT) }\end{array}$ & $\begin{array}{c}\text { Samples } \\
(N)\end{array}$ \\
\hline \multicolumn{7}{|l|}{ Hole 896A: } \\
\hline $195<$ & All & $17.6 \pm 10.6$ & $7.8 \pm 5.1$ & $-4.3 \pm 20.8$ & $17.1 \pm 11.2$ & 166 \\
\hline \multirow[t]{3}{*}{$<460$} & Massive & $22.3 \pm 11.2$ & $6.3 \pm 6.3$ & $-3.5 \pm 24.4$ & $11.2 \pm 6.2$ & 77 \\
\hline & Pillow & $0.0 \pm 0.0$ & $0.0 \pm 0.0$ & $0.0 \pm 0.0$ & $0.0 \pm 0.0$ & 0 \\
\hline & Breccia & $30.7 \pm 8.7$ & $2.4 \pm 1.9$ & $5.5 \pm 65.8$ & $12.0 \pm 11.3$ & 2 \\
\hline $195<$ & All & $9.1 \pm 4.0$ & $11.2 \pm 4.2$ & $-9.2 \pm 10.5$ & $26.2 \pm 11.8$ & 75 \\
\hline \multirow{2}{*}{$<330$} & Massive & $11.9 \pm 3.0$ & $11.4 \pm 3.7$ & $-8.1 \pm 7.6$ & $18.1 \pm 5.9$ & 26 \\
\hline & Pillow & $0.0 \pm 0.0$ & $0.0 \pm 0.0$ & $0.0 \pm 0.0$ & $0.0 \pm 0.0$ & 0 \\
\hline $330<$ & All & $21.9 \pm 4.2$ & $8.0 \pm 4.1$ & $-4.6 \pm 8.9$ & $11.6 \pm 4.5$ & 40 \\
\hline \multirow[t]{2}{*}{$<360$} & Massive & $23.2 \pm 4.5$ & $5.7 \pm 3.4$ & $-4.3 \pm 7.5$ & $8.5 \pm 1.8$ & 18 \\
\hline & Pillow & $0.0 \pm 0.0$ & $0.0 \pm 0.0$ & $0.0 \pm 0.0$ & $0.0 \pm 0.0$ & 0 \\
\hline $360<$ & All & $26.8 \pm 11.0$ & $3.1 \pm 2.4$ & $2.8 \pm 33.1$ & $10.7 \pm 5.6$ & 51 \\
\hline \multirow[t]{3}{*}{$<460$} & Massive & $29.6 \pm 11.5$ & $3.2 \pm 2.8$ & $0.2 \pm 35.7$ & $8.7 \pm 4.8$ & 33 \\
\hline & Pillow & $0.0 \pm 0.0$ & $0.0 \pm 0.0$ & $0.0 \pm 0.0$ & $0.0 \pm 0.0$ & 0 \\
\hline & Breccia & $30.7 \pm 8.7$ & $2.4 \pm 1.9$ & $5.5 \pm 65.8$ & $12.0 \pm 11.3$ & 2 \\
\hline Hole 504B: & & $26.2 \pm 13.8$ & $6.2 \pm 5.5$ & $-22.4 \pm 20.4$ & $13.5 \pm 7.5$ & \\
\hline Above Unit 2D & $11.7 \pm 5.8$ & $11.2 \pm 5.0$ & $-10.8 \pm 6.0$ & $25.4 \pm 13.7$ & & \\
\hline Unit 2D and below & $27.7 \pm 13.5$ & $5.7 \pm 5.3$ & $-23.7 \pm 21.0$ & $12.3 \pm 5.5$ & & \\
\hline
\end{tabular}

Note: Errors are given as 1 standard deviation. $N=$ number of samples.

probably indicates that hematite is a product of inversion. Type I behavior was observed throughout the hole, in both massive and pillow lithologies.

Type II curves are nearly reversible in shape, though not in magnitude, with a single peak matched by a similar cooling curve (Fig. $4 \mathrm{E})$. These curves can be interpreted as an indication of the presence of titanomagnetite. The majority of the rype II curves occur in samples taken from below 330 mbsf (above 330 mbsf, only Sample 148$896 \mathrm{~A}-3 \mathrm{R}-1,68-71 \mathrm{~cm}$, shows type II behavior), in both pillow and massive flows.

Almost all the samples, of both types, show a bulge on the heating curve, also commonly seen on the cooling curve, in the interval between $50^{\circ}$ and $200^{\circ} \mathrm{C}$. The apparent reversibility of this section is characteristic of a mineral that is chemically stable up to $700^{\circ} \mathrm{C}$, and may represent a component of original titanomagnetite. The Curie temperature of titanomagnetites is highly dependent on the composition, particularly of cations such as aluminum or magnesium replacing titanium (O'Reilly, 1984). Previous studies of the oxide compositions of the extrusive rocks from DSDP Hole 504B (Donovan and O'Reilly, 1983) have determined $x$ values of about 0.6 for titanomagnetites with a general formula of $\mathrm{Fe}_{3-d-x} \mathrm{M}_{d} \mathrm{Ti}_{x} \mathrm{O}_{4}$, where $\mathrm{M}$
$=\mathrm{Al}, \mathrm{Mg}, \mathrm{Mn}$, and $\mathrm{Cr}$. The minor cations, principally $\mathrm{Al}$, have $d$ values of about 1.6, sufficient to significantly effect the Curie temperature (Ozdemir and O'Reilly, 1981). Despite this, the temperature interval of the bulge between $50^{\circ}$ and $200^{\circ} \mathrm{C}$ is consistent with a stoichiometric titanomagnetite with $x=0.6\left(T_{c}=150^{\circ} \mathrm{C}\right)$. This phase may thus represent a small component of unoxidized original titanomagnetite.

For most samples, the heating curve shows little susceptibility above $600^{\circ} \mathrm{C}$, although some of these samples show a gradual rise in susceptibility on the cooling sample between $700^{\circ}$ and $600^{\circ} \mathrm{C}$, which is characteristic of hematite generated during heating. Genuinely reversible behavior in the $600^{\circ}-700^{\circ} \mathrm{C}$ interval was identified only in three samples (see Table 3 ).

The following conclusions can be drawn from the thermomagnetic behavior: the majority of samples show evidence of the typical thermomagnetic behavior ascribed to titanomaghemite and inversion to multiphase products (e.g., Donovan and O'Reilly, 1983). Some samples show thermomagnetic behavior typical of titanomagnetites. Type I and II behavior occurs throughout the hole, although type II behavior is most common below $330 \mathrm{mbsf}$, and there is no clear change in the inversion temperature or of the peak temperature of the 
Table 3. Measurements of susceptibility at high temperature, Hole 896A.

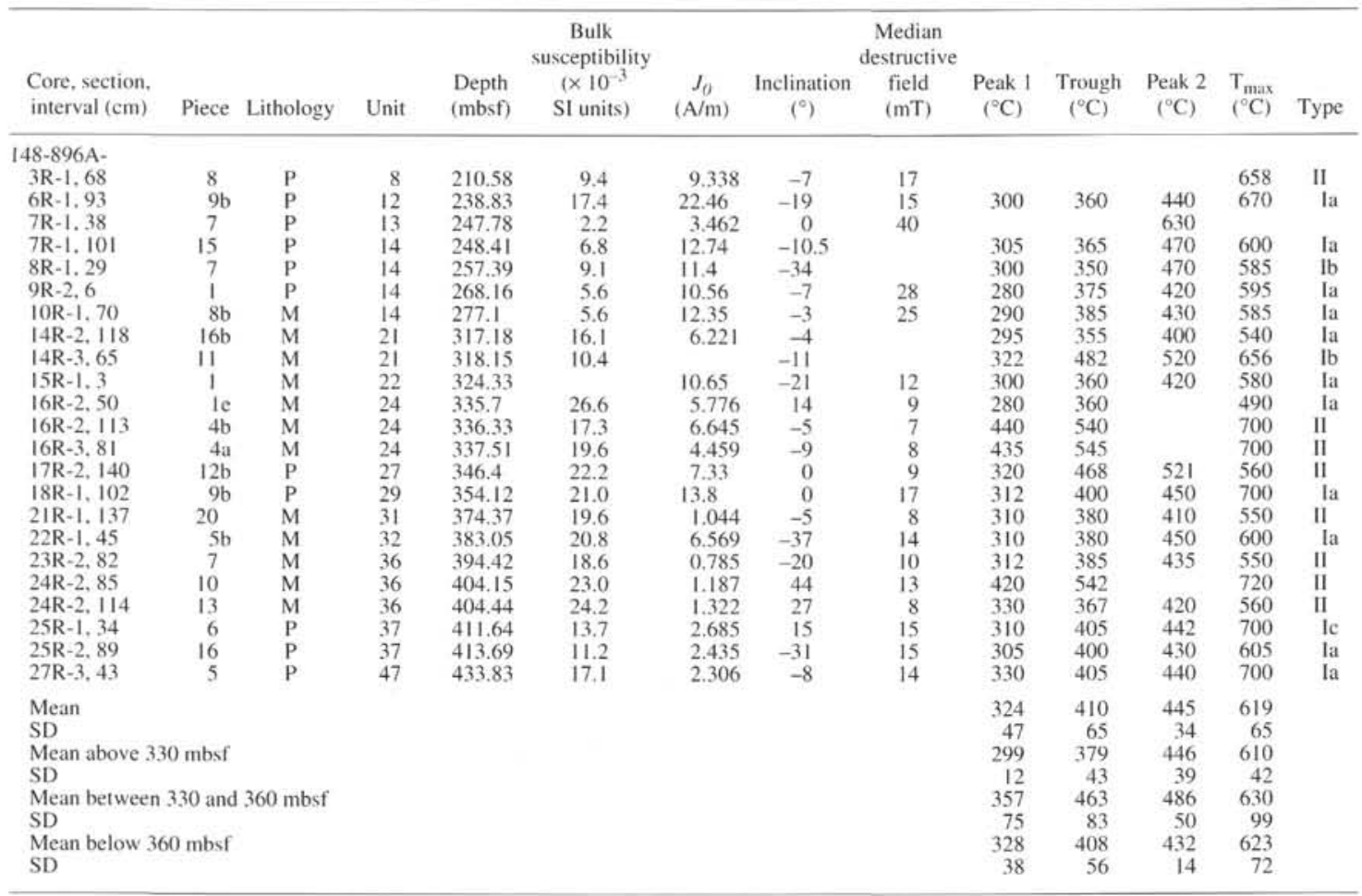

Notes: Peak $1=$ temperature of the first peak; Trough = temperature of the trough between the two peaks: Peak $2=$ temperature of the second peaks; and $T_{\text {max }}=$ temperature at which the susceptibility drops to zero. Type is defined in the text. SD $=1$ standard deviation.

product with depth. The absence of change in these temperatures, which are highly sensitive to the ratio of $\mathrm{Fe}$ to $\mathrm{Ti}$ and other cations, suggests that there is little change in the relative abundance of the cations, although the increase in the occurrence of type II behavior may be related to subtle changes in the stoichiometry of the iron oxides.

\section{MAGNETIC MINERALOGY}

The core recovered in Hole $896 \mathrm{~A}$ can be separated into upper, middle, and lower sections that exhibit different magnetic properties. The first boundary, between the upper and middle sections (at about $330 \mathrm{mbsf}$ ), is marked by a distinct rise in susceptibility (Fig. 3). The second boundary, between the middle and lower sections (at about $360 \mathrm{mbsf}$ ), is based on a decrease in $J_{0}$, a higher scatter in the stable magnetic inclinations, and an increase in the maximum susceptibility recorded downhole. The MDF appears to decrease more gradually downhole. The upper section (above $330 \mathrm{mbsf}$ ) has high $J_{0}$ values $(11.2 \pm 4.2 \mathrm{~A} / \mathrm{m})$, high magnetic stability $(\mathrm{MDF}=26.2 \pm 11.8 \mathrm{mT})$, and low bulk susceptibilities $\left(9.1 \pm 4.0 \times 10^{-3} \mathrm{SI}\right.$ units). The middle section (between 330 and $360 \mathrm{mbsf}$ ) is bounded at the top by the massive flow Unit $24 . J_{0}$ is high $(8.0 \pm 4.1 \mathrm{~A} / \mathrm{m})$, but magnetic stability (MDF) is low $(11.6 \pm 4.5 \mathrm{mT})$ and susceptibility is high $(21.9 \pm 4.2$ $\times 10^{-3} \mathrm{SI}$ units). The magnetic remanence is characterized by single stable magnetic components in both the upper and middle sections. The lower section (below $360 \mathrm{mbsf}$ to the bottom of the hole at 469 mbsf) is bounded at the top by the first of a package of massive flows (Units $31-36)$. In this section $J_{0}$ is low $(3.1 \pm 2.4 \mathrm{~A} / \mathrm{m}), \mathrm{MDF}$ is low $(10.7 \pm 5.6 \mathrm{mT})$, and the susceptibility is high and variable $(26.8 \pm$ $11.0 \times 10^{-3} \mathrm{SI}$ units). The magnetic components show great circle trajectories, indicating overprinting, and the inclinations show a high degree of scatter.

The transitions between these sections (at 330 and $360 \mathrm{mbsf}$ ) do not correspond to the bulk change in petrology observed in the core; the change between plagioclase-olivine phyric basalt above to plagioclase-olivine-clinopyroxene phyric basalt below occurs at about 390 mbsf. There is, however, a distinct change in the $\mathrm{TiO}_{2}$ content, and a more subtle change in $\mathrm{Fe}_{2} \mathrm{O}_{3}$, at a depth of about $345 \mathrm{mbsf}$ (see Alt, Kinoshita, Stokking, et al., 1993). This geochemical change is also reflected in other elemental compositions, including $\mathrm{SiO}_{2}$ and $\mathrm{CaO}$.

The saturation remanence $\left(J_{r s}\right)$ shows a variation downhole similar in form to that of $\mathrm{Fe}_{2} \mathrm{O}_{3}$. This parameter probably represents a good estimate of the amount of ferrous oxide in the basalt, and it varies by a factor of about $20 \%$. This suggests that the actual concentration of the oxide phase does not contribute significantly to the variation in magnetization, which is much larger.

Another important control on the magnetic properties is the grain size. This property is difficult to measure directly, because the opaque grains in the fine-grained or microcrystalline groundmass are in general below optical resolution. We have attempted to quantify the effect of grain-size variation by estimating the average grain size from one property (MDF) assuming that the concentration and composition are invariant, and then using this to generate an estimate of the other properties, which can be compared with the actual measurements. This is similar to the approach adopted by O'Reilly et al. (1993), although they used more complex parameters, including a simultaneous estimate of the degree of maghematization and grain size. We do not think that this is appropriate for this data set because we have not measured the degree of maghematization and because this model is appropriate only for a narrow range of compositions. The grain size of the magnetites has been related to the hysteresis properties, particularly the coercive force, $H_{r}$ (Heider et al., 1987). This relationship is not entirely suitable for titanomagnetites (see, e.g., fig. 7.9 in O'Reilly, 1984), but it does give an indication of the variation in grain size, though not any absolute values. Although we do not have direct measurements of the coercive force for samples from this hole, Furuta (1983) showed that there is a strong correlation 

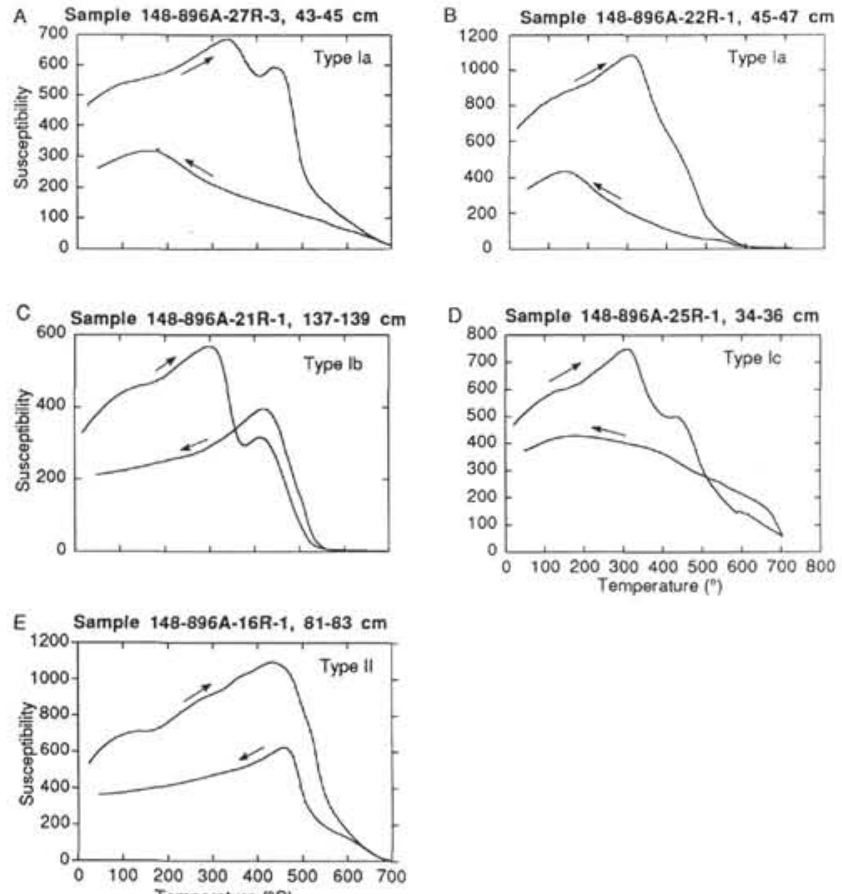

Figure 4. Bulk susceptibility vs. temperature. Heating and cooling runs are indicated by arrows. A. Type la irreversible thermomagnetic curve with well-defined first peak, trough, and second peak. The cooling curve shows a gradual slope, rising to a peak at about $200^{\circ} \mathrm{C}$. B. Type la irreversible thermomagnetic curve with well-defined first peak, steep slope, first point of inflection (corresponding to the trough), shallower slope, second point of inflection (corresponding to the second peak), and steeper slope. The cooling curve shows a gradual slope, rising to a peak at about $200^{\circ} \mathrm{C}$. C. Type Ib irreversible thermomagnetic curve. The cooling curve has a steep slope, with a peak at about $500^{\circ} \mathrm{C}$. D. Type Ic irreversible thermomagnetic curve. The cooling curve rises between $600^{\circ}$ and $700^{\circ} \mathrm{C}$. E. Type II partially reversible thermomagnetic curve, reaching a single peak at about $450^{\circ} \mathrm{C}$. The cooling curve also shows a peak at about $460^{\circ} \mathrm{C}$, although with a lower susceptibility. Note that all curves have a bulge on their heating slopes between about $30^{\circ}$ and $200^{\circ} \mathrm{C}$, which is also visible on some of the cooling curves.

between $H_{c}$ and MDF. We have used this correlation to estimate $H_{t}$ from the MDF of samples from Hole 896A, and thus to estimate the grain size of samples (Fig. 5A). We can go further, and test to what extent $J_{0}$ and susceptibility can be explained by a simple variation in grain size. The variation of susceptibility with grain size has been discussed by O'Reilly (1984) for different compositions, including $x=$ 0.61 , which we have chosen to use. From this variation we can predict the variation in susceptibility (although not any absolute values). This estimate is shown in Figure 5B. The strong correlation between the estimated and measured values of susceptibility (Fig. 5D) suggests that a large part of the variability in this parameter is related to changes in grain size without any need to evoke significant variability in magnetic mineralogy or concentration of magnetic grains. This is supported by the correlation between susceptibility and the log of MDF (Fig. 5F), which from the model of O'Reilly et al. (1993) would be expected if the variation in the degree of maghematization and the concentration are insignificant.

We can also estimate $J_{0}$ assuming that the magnetization is similar to a thermo-remanent magnetization (TRM) (if the magnetic mineral is maghemite, this case is not likely; however, Thellier-Thellier experiments [S. Allerton, unpubl. data] suggest that the magnetization may mimic a TRM), using the parameters published by Dunlop
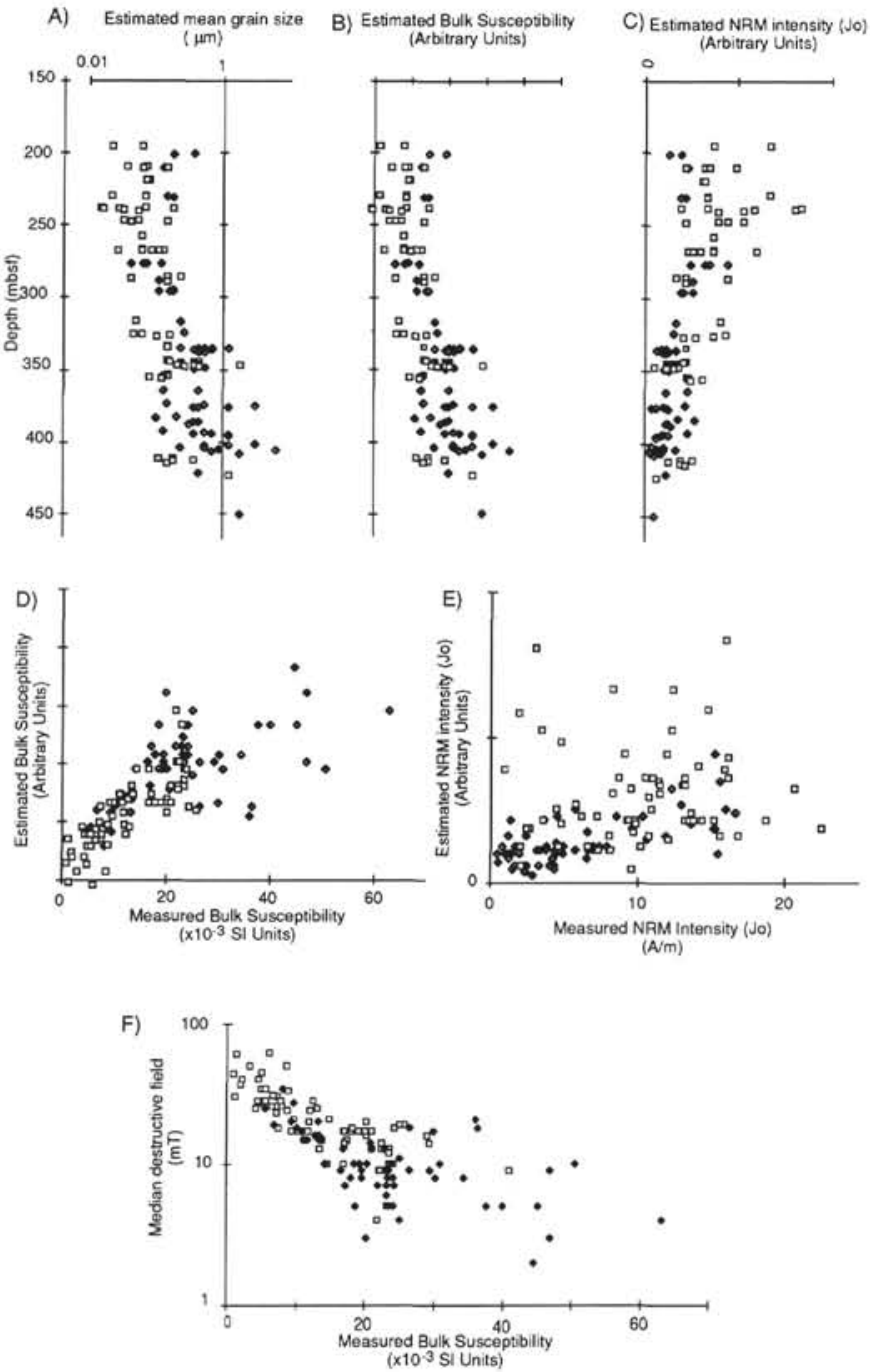

Figure 5. A. Estimate of the grain size of magnetic grains vs. depth. B. Bulk susceptibility estimated from grain size vs. depth. C. $J_{0}$ estimated from grain size vs. depth. D. Correlation of estimated and measured bulk susceptibility. E. Correlation of estimated and measured $J_{0}$. F. MDF plotted on a log scale vs. bulk susceptibility.

(1981). This estimate (Fig. 5C, E) can explain some of the general features of the curve - the high magnetization in the upper part of the hole and lower magnetization in the lower part. The estimate does predict some particularly high values of $J_{0}$ in the middle of the upper part of the hole that were not observed. Other factors will control $J_{0}$, particularly the degree of maghematization and the magnetization process (for example, thermal or chemical remanence), so it is perhaps not surprising that $J_{0}$ cannot be modeled by grain size alone.

Evidence for hematite from reversible thermomagnetic behavior in the $600^{\circ}$ to $700^{\circ} \mathrm{C}$ range and from high values of $H_{\text {sat }}$ from IRM acquisition are restricted largely to the thick massive flow Units 21,24, and 36. These exhibit high levels of pervasive alteration, and hematite has been reported in thin section from Unit 36 (Alt, Kinoshita, Stokking, et al., 1993).

Furuta and Levi (1983) suggested that the largest control on magnetic properties in Hole 504B is lithologic, related to grain size, with high $J_{0}$ and low susceptibilities in pillow flows and low $J_{0}$ and high susceptibilities in massive flows. The results of this study indicate that within each of the sections identified in Hole 896A, the massive 
flows and pillow flows have slightly different properties, but that the differences in the properties of pillow flows and massive flows between the sections is greater than that between the types of flows in any individual section.

\section{COMPARISON WITH HOLE 504B}

The magnetic properties of the upper part of the extrusive sequence of Hole 504B have been described by Furuta and Levi (1983) (see Table 2). The first $39 \mathrm{~m}$ of the basement of Hole 504B, to the top of massive flow Unit 2D ( $313 \mathrm{mbsf}$ ), has many properties in common with the first $135 \mathrm{~m}$ of basement in Hole $896 \mathrm{~A}$ (see Table 2). For example, $J_{0}$ in Hole $504 \mathrm{~B}$ is $11.2 \pm 5.0 \mathrm{~A} / \mathrm{m}$, compared to $11.2 \pm 4.2$ $\mathrm{A} / \mathrm{m}$ in Hole $896 \mathrm{~A}$, and the bulk susceptibility in Hole 504B is 11.7 $\pm 5.8 \times 10^{-3}$ SI units, compared to $13.8 \pm 5.6 \times 10^{-3}$ SI units in Hole 896A. The stable inclinations are also similar: $-10.8^{\circ} \pm 6.0^{\circ}$ in Hole 504B compared to $-9.2^{\circ} \pm 10.5^{\circ}$ in Hole $896 \mathrm{~A}$. Both of these values are close to the expected paleoinclination $\left(-7^{\circ}\right)$, suggesting that there has been little tilting of this part of the sequence. The base of these sequences is at $3773 \pm 3 \mathrm{mbsl}$ in Hole $504 \mathrm{~B}$ and at $3796 \pm 3 \mathrm{mbsl}$ in Hole 896A (see Fig. 6). Comparison of the geochemistry of these two holes shows that they both show an increase in $\mathrm{TiO}_{2}$ and a decrease in $\mathrm{Al}_{2} \mathrm{O}_{3}$ and in $\mathrm{Cr}$ at about this level. It does not necessarily follow that these upper extrusive rocks are from identical flows or volcanic sources, but it is likely that they are of a similar origin, post-dating the flow beneath.

Below 313 mbsf in Hole 504B, basement has properties similar to the lower section of Hole 896A (see Table 2). For example, in Hole 504B the bulk susceptibility is $27.7 \pm 13.5 \times 10^{-3}$ SI units, whereas it is $26.8 \pm 11.0 \times 10^{-3} \mathrm{SI}$ units in Hole $896 \mathrm{~A} . J_{0}$ is $5.7 \pm 5.3 \mathrm{~A} / \mathrm{m}$ in Hole $504 \mathrm{~B}$ and $3.1 \pm 2.4 \mathrm{~A} / \mathrm{m}$ in Hole $896 \mathrm{~A}$. The inclinations in Hole 504B below $313 \mathrm{mbsf}$ are $-23.7^{\circ} \pm 21.0^{\circ}$, which Furuta and Levi (1983) have related to a tilting of the extrusive rocks away from the axis.

\section{IMPLICATIONS FOR THE EVOLUTION OF SITES 504 AND 896}

The upper sections of Holes 504B and 896A have features that suggest that they were extruded slowly, a few kilometers away from the ridge axis, onto an approximately level, perhaps ponded, flow surface, and that the section below was extruded close to the axis. lows:

The reasons for making this assertion can be summarized as fol-

1. The very fine grain sizes indicated by the magnetic stability may result from slow effusion, which allows rapid cooling.

2. If the apparently cyclical nature of the magnetic inclinations in the upper section results from secular variation, then extrusion occurred gradually over a few thousand years.

3. The inclinations of the upper sections of Holes 504B and 896A are very close to the stable reference inclination, suggesting that the upper section has not been tilted. In Hole 504B, the section below has been tilted, suggesting that these sections are separated by an unconformity.

4. Pervasive alteration is much more extensive in the lower part of the section (Alt, Kinoshita, Stokking, et al., 1993). This suggests that either the upper section was extruded after the main phase of hydrothermal circulation was over, or that the massive flow unit acted as an effective seal to hydrothermalism beneath. Pezard (1990) has emphasized the importance of Unit 2D in Hole 504B as a hydrological barrier, from various downhole logs and from packer and temperature measurements.

5. The base of the upper sections is at $3773 \pm 3 \mathrm{mbsl}$ in Hole $504 \mathrm{~B}$ and at $3793 \pm 3 \mathrm{mbsl}$ in Hole $896 \mathrm{~A}$, so the upper sequence was built

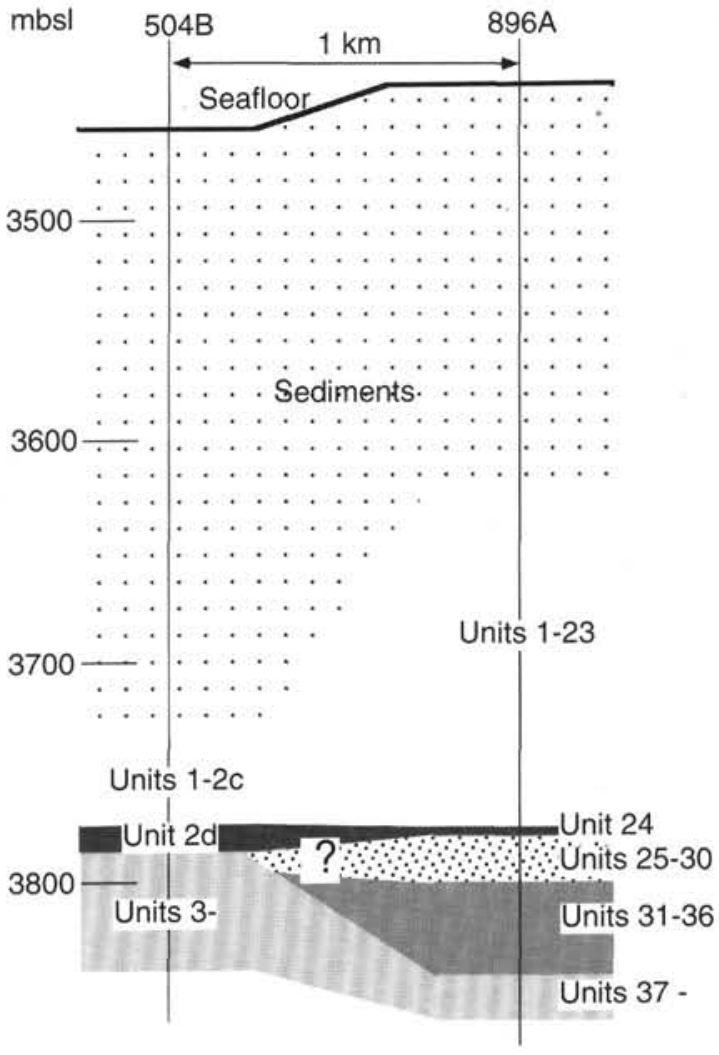

Figure 6. Cartoon illustrating the correlation between Hole 504B and Hole $896 \mathrm{~A}$ based on magnetic properties.

up on an approximately level top surface of a massive flow (Fig. 6). The apparent dip of this surface between the two holes is about $2^{\circ}$. The thickness of this flow unit is between 24.0 and $10.4 \mathrm{~m}$ in Hole 504B and between 9.1 and $3.6 \mathrm{~m}$ in Hole 896A, consistent with ponding of the flow.

\section{CONCLUSIONS}

The basement of Hole $896 \mathrm{~A}$ can be divided into three sections on the basis of its magnetic properties (see Table 2 and Fig. 3). The upper section, to $330 \mathrm{mbsf}$, has relatively high $J_{0}$ and low susceptibility values. The middle section, between 330 and $360 \mathrm{mbsf}$, has a high $J_{0}$ and intermediate susceptibility values, and the lower section, below $360 \mathrm{mbsf}$, has low $J_{O}$ and high susceptibility values. The magnetic stability and susceptibility can be related to the size of the magnetic grains. The values of $J_{0}$ can be modeled by grain size in a broad sense, but in detail the effects of the maghematization and magnetization process probably also control the intensity of magnetization.

The magnetic properties of the basement of Hole $896 \mathrm{~A}$ can be correlated with those of Hole 504B. The base of the upper section corresponds to the massive flow Unit 24 in Hole 896A and to the massive flow Unit 2D in Hole 504B. The apparent dip of this level between the two holes is about $2^{\circ}$. The upper section probably represents an off-axis lava pile with an irregular upper surface topography that was extruded over a relatively long period of time. The massive flow unit at its base apparently acted as an important barrier to hydrothermal fluids, focusing alteration beneath it.

These results have significant implications for the interpretation of magnetic field data from ridges. The uppermost layer of late extrusive rocks has a high magnetization and a highly variable thickness; 
it may be an important contributor to variations in the magnetic signal at ridges. Thus, magnetic segmentation (Sempere, 1991) may result from the increased thickness of late extrusive rocks rather than from variations in the magnetization of the basalt sequence as a whole.

\section{ACKNOWLEDGEMENTS}

We would like to thank W. O'Reilly, S. Beske-Diehl, and Carl Richter for providing thoughtful and constructive reviews of this paper. Particular thanks go to M. Halstedt for her advice and assistance on the JOIDES Resolution.

\section{REFERENCES}

Ade-Hall, J.M., and Johnson, H.P., 1976. Paleomagnetism of basalts, Leg 34. In Yeats, R.S., Hart, S.R., et al., Init. Repts. DSDP, 34: Washington (U.S. Govt. Printing Office), 513-532.

Allerton, S., Pariso, J.E., Stokking, L.B., and McClelland, E., 1995. Origin of the natural remanent magnetism of sheeted dikes in Hole $504 \mathrm{~B}$ cored during Legs 137 and 140. In Erzinger, J., Becker, K., Dick, H.J.B., and Stokking, L.B. (Eds.), Proc. ODP. Sci. Results, 137/140: College Station, TX (Ocean Drilling Program), 263-270.

Alt, J.C., Kinoshita, H., Stokking, L.B., et al., 1993. Proc. ODP. Init. Repts., 148: College Station, TX (Ocean Drilling Program).

Banerjee, S.K., 1980. Magnetism of the oceanic crust: evidence from ophiolite complexes. J. Geophys. Res., 85:3556-3566.

Cande, S.C., and Kent, D.V., 1992. A new geomagnetic polarity time scale for the Late Cretaceous and Cenozoic. J. Geophys. Res., 97:1391713951.

Donovan, J.B., and O'Reilly, W., 1983, Magnetic properties of basalts from Hole 504B, Deep Sea Drilling Project Leg 69. In Cann, J.R., Langseth. M.G., Honnorez, J., Von Herzen, R.P., White, S.M., et al., Init. Repts. DSDP, 69: Washington (U.S. Govt. Printing Office), 721-726.

Dunlop, D.J., 1981. The rock magnetism of fine particles. Phys. Earth Planet. Inter., 26:1-26.

Furuta, T., 1983. Magnetic properties of basalt samples from Holes 504B and 505B on the Costa Rica Rift, Deep Sea Drilling Project Legs 69 and 70. In Cann, J.R., Langseth, M.G., Honnorez, J., Von Herzen, R.P., White, S.M., et al., Init. Repts. DSDP, 69: Washington (U.S. Govt. Printing Office), 711-720.

Furuta, T., and Levi, S., 1983. Basement paleomagnetism of Hole 504B. In Cann, J.R., Langseth, M.G., Honnorez, J., Von Herzen, R.P.. White. S.M., et al., Init. Repts. DSDP, 69: Washington (U.S. Govt. Printing Office), 697-703.

Heider, F., Dunlop, D.J., and Sugiura, N., 1987. Magnetic properties of hydrothermally recrystallized magnetite crystals. Science, 236:12871290.

Hobart, M.A., Langseth, M.G., and Anderson, R.N., 1985. A geothermal and geophysical survey on the south flank of the Costa Rica Rift: Sites 504 and 505. In Anderson, R.N., Honnorez, J., et al., Init. Repts. DSDP, 83: Washington (U.S. Govt. Printing Office), 379-404.

Johnson, H.P., 1978. Paleomagnetism of igneous rock samples-DSDP Leg 45. In Melson, W.G., Rabinowitz, P.D., et al., Init. Repts. DSDP, 45: Washington (U.S. Govt. Printing Office), 387-396.

Kirschvink, J.L., 1980. The least-squares line and plane and analysis of palaeomagnetic data. Geophys. J. R. Astron. Soc., 62:699-718.

LaBrecque, J.L., Kent. D.V., and Cande, S.C., 1977. Revised magnetic polarity time scale for Late Cretaceous and Cenozoic time. Geology, 5:330-335.

Langseth, M.G., Mottl, M.J., Hobart, M.A., and Fisher, A., 1988. The distribution of geothermal and geochemical gradients near Site 501/504: implications for hydrothermal circulation in the oceanic crust. In Becker, K., Sakai, H., et al., Proc. ODP. Init. Repts., 111: College Station, TX (Ocean Drilling Program), 23-32.

Lowrie, W., and Kent, D.V.. 1978. Characteristics of VRM in ocean basalts. J. Geophys., 44:297-315.

O'Reilly, W., 1984. Rock and Mineral Magnetism: New York (Chapman and Hall).

O'Reilly, W., Brown, K., Styles, P., and Bloxam, T.W., 1993. A detailed geochemical and rock magnetic study of dredged basalt from the Sheba Ridge, Gulf of Aden. Mar. Geophys. Res., 15:101-119.

Ozdemir, O., and O'Reilly, W., 1981. Laboratory synthesis of aluminum-substituted titanomaghemites and their characteristic properties. $J$. Geophys., 49:93-100.

Pariso, J.E., and Johnson, H.P., 1991. Alteration processes at Deep Sea Drilling Project/Ocean Drilling Program Hole 504B at the Costa Rica Rift: implications for magnetization of oceanic crust. J. Geophys. Res., 96: 11703-11722.

Pechersky, D.M., Tikhonov, L.V., and Pertsev, N.N., 1983. Magnetic properties of basalts, Deep Sea Drilling Project Legs 69 and 70. In Cann, J.R., Langseth, M.G., Honnorez, J., Von Herzen, R.P., White, S.M., et al., Init. Repts. DSDP, 69: Washington (U.S. Govt. Printing Office), 705-710.

Pezard, P.A., 1990. Electrical properties of mid-ocean ridge basalt and implications for the structure of the upper oceanic crust in Hole 504B. J. Geophys. Res., 95:9237-9264.

Sempere, J.-C., 1991. High-magnetization zones near spreading center discontinuities. Earth Planet. Sci. Lett., 107:389-405.

Smith, G.M., and Banerjee, S.K., 1986. Magnetic structure of the upper kilometer of the marine crust at Deep Sea Drilling Project Hole 504B, Eastern Pacific Ocean. J. Geophys. Res., 91:10337-10354.

Vine, F.J., and Moores, E.M., 1972. A model for the gross structure, petrology, and magnetic properties of oceanic crust. Mem.-Geol. Soc. Am. 132:195-205.

Date of initial receipt: 15 August 1994 Date of acceptance: 24 February 1995 Ms 148SR-129 